\title{
Quantitative determination of vortex core dimensions in headtohead domain walls using offaxis electron holography
}

Junginger, F; Klaui, M; Backes, D; Krzyk, S; Rüdiger, U; Kasama, T; Dunin-Borkowski, Rafal E.; Feinberg, JM; Harrison, RJ; Heyderman, LJ

Published in:

Applied Physics Letters

Link to article, DOI:

$10.1063 / 1.2829601$

Publication date:

2008

Document Version

Publisher's PDF, also known as Version of record

Link back to DTU Orbit

Citation (APA):

Junginger, F., Klaui, M., Backes, D., Krzyk, S., Rüdiger, U., Kasama, T., Dunin-Borkowski, R. E., Feinberg, JM., Harrison, RJ., \& Heyderman, LJ. (2008). Quantitative determination of vortex core dimensions in headtohead domain walls using offaxis electron holography. Applied Physics Letters, 92(11), 112502. https://doi.org/10.1063/1.2829601

\section{General rights}

Copyright and moral rights for the publications made accessible in the public portal are retained by the authors and/or other copyright owners and it is a condition of accessing publications that users recognise and abide by the legal requirements associated with these rights.

- Users may download and print one copy of any publication from the public portal for the purpose of private study or research.

- You may not further distribute the material or use it for any profit-making activity or commercial gain

- You may freely distribute the URL identifying the publication in the public portal 


\title{
Quantitative determination of vortex core dimensions in head-to-head domain walls using off-axis electron holography
}

\author{
F. Junginger, ${ }^{\text {a) }}$ M. Kläui , ${ }^{\text {b) }}$ D. Backes, ${ }^{\text {c) }}$ S. Krzyk, and U. Rüdiger \\ Fachbereich Physik, Universität Konstanz, Universitätsstraße 10, 78457 Konstanz, Germany \\ T. Kasama and R. E. Dunin-Borkowski ${ }^{\text {d) }}$ \\ Department of Materials Science and Metallurgy, University of Cambridge, Pembroke Street, \\ Cambridge CB2 3QZ, United Kingdom \\ J.-M. Feinberg ${ }^{\mathrm{e})}$ and R. J. Harrison \\ Department of Earth Sciences, University of Cambridge, Pembroke Street, Cambridge CB2 3QZ, \\ United Kingdom \\ L. J. Heyderman \\ Laboratory for Micro- and Nanotechnology, Paul Scherrer Institut, 5232 Villigen PSI, Switzerland
}

(Received 12 October 2007; accepted 7 December 2007; published online 17 March 2008)

In this paper, we present a complete three-dimensional characterization of vortex core spin structures, which is important for future magnetic data storage based on vortex cores in disks and in wires. Using electron holography to examine vortices in patterned Permalloy devices we have quantitatively measured the in-plane and out-of-plane magnetization of a vortex core. Observed core widths and integrated phase shifts agree well with those derived from micromagnetic simulations.

(C) 2008 American Institute of Physics. [DOI: 10.1063/1.2829601]

Confined magnetic structures have become the recent focus of intense research since they exhibit fundamental physical effects and might also prove useful for applications. ${ }^{1}$ One of the smallest confined magnetic structures is the singularity in the center of a curled vortex spin configuration, which points out of plane. This so-called "vortex core" has lateral dimensions, which are determined by the interplay between the exchange energy and the stray field energy. ${ }^{2}$ In materials consisting of $3 d$ metals, the core's lateral dimensions are of the order of a few nanometers. ${ }^{2}$ Vortices in disk structures have been widely studied using magnetic force microscopy but, due to their small dimensions, only qualitative observations of vortex cores have been achieved. ${ }^{3}$ The only quantitative observations of vortex core dimensions come from studies using spin-polarized scanning tunneling microscopy (SP-STM) for single crystal Fe. ${ }^{2,4}$ This technique only works for very smooth structures in ultrahigh vacuum and is not well suited for studying ex situ patterned devices made of polycrystalline materials such as $\mathrm{Ni}_{80} \mathrm{Fe}_{20}$ alloy (Permalloy).

Measurements of the vortex core dynamics in Permalloy have recently revealed potentially useful magnetic behavior. Due to its topology, a vortex core moves perpendicular to the direction of the force acting on it. ${ }^{5}$ Such a gyrotropic motion then leads to a spiralling motion of the core after it has been excited by a field pulse and a circular motion if a resonant ac excitation is used. ${ }^{6}$ The direction of the motion is given purely by the out-of-plane component of the vortex core regardless of whether the magnetization curls clockwise or

\footnotetext{
a) Also at: Department of Materials Science and Metallurgy, University of Cambridge, Pembroke Street, Cambridge CB2 3QZ, UK.

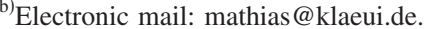

${ }^{c}$ Also at: Laboratory for Micro- and Nanotechnology, Paul Scherrer Institut, 5232 Villigen PSI, Switzerland.

d) Also at: Center for Electron Nanoscopy, DTU, DK-2800 Denmark.

e) Also at: Institute for Rock Magnetism, University of Minnesota, Minneapolis, MN 55455.
}

counterclockwise around the core. Vortex core polarity has also been proposed as the basis for information storage and to reliably switch a vortex core's polarity large fields are necessary in static experiments. One particular exciting polarity switching technique has been recently reported, where the vortex core in Permalloy structures is reversed with short bursts of small fields. ${ }^{6}$ An alternative polarity switching mechanism uses the current-induced spin torque effect, which has the additional advantage of not requiring fieldgenerating strip lines. In general spin polarized currents are predicted to displace domain walls and induce domain wall transformations. ${ }^{7}$ Recent experimental reports confirm these predictions of domain wall movement ${ }^{8-10}$ and transformations. ${ }^{11}$ However, the details of this domain wall behavior are still poorly understood. In thin film wires, the two domain wall types that occur are transverse and vortex walls and the latter contains a vortex core. ${ }^{12}$ To properly explore this magnetic behavior, in particular the transformations to and from the vortex walls, an imaging technique is required that provides an accurate determination of the vortex's spin structure and polarity. Off-axis electron holography is a powerful method for studying the in-plane component of magnetic structures. ${ }^{13}$ Although the idea of tilting a sample in the transmission electron microscope (TEM) to get a three-dimensional induction distribution already existed, ${ }^{14}$ no quantitative imaging of the out-of-plane spin structure of a vortex domain wall has been reported. In this paper, we present the results of electron holography observations of the in-plane and out-of-plane components of a vortex core in a polycrystalline Permalloy nanowire. We deduce the vortex core width from the out-of-plane and in-plane magnetization distribution and compare the results to both analytical and numerical simulations.

The ferromagnetic samples used here are fabricated from Permalloy by electron-beam lithography ${ }^{15}$ on a silicon nitride membrane. The shape of the specimen is a wavy line with lateral constrictions (notches), which pin the domain 
(a)
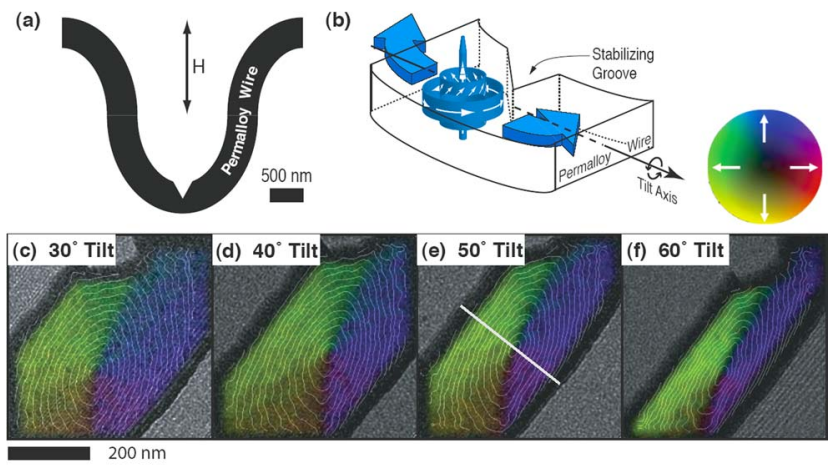

FIG. 1. (Color online) The layout of the Permalloy nanowire with the magnetization direction of the external field to induce the vortex domain wall next to the notch is shown schematically in (a) and the resulting magnetization configuration is presented schematically in (b). Electron holography images of the projected magnetic components of a vortex domain wall in a Permalloy wire $\left(440 \times 40 \mathrm{~nm}^{2}\right)$ at different tilt angles are shown [(c) $30^{\circ}$, (d) $40^{\circ}$, (e) $50^{\circ}$, and (f) $\left.60^{\circ}\right]$ revealing the curled in-plane magnetization components.

walls [Fig. 1(a)]. ${ }^{16}$ In the region of interest, the sample is $40 \pm 4 \mathrm{~nm}$ thick and the structure is around $440 \mathrm{~nm}$ wide.

In order to investigate the theoretical properties of magnetic vortices in Permalloy, we calculated micromagnetic simulations of vortices within a $1000 \times 1000 \times 40 \mathrm{~nm}^{3}$ volume using the Landau-Liftshitz-Gilbert equations of motion. ${ }^{17}$ Simulations used a finite grid of 2 or $5 \mathrm{~nm}$ cubes, which are sufficiently fine to treat the gross geometry of vortices within Permalloy. ${ }^{18}$ Each simulation includes a $75 \mathrm{~nm}$ thick layer of deactivated elements above and below the modeled Permalloy layer to allow for the inclusion of stray fields when calculating integrated phase images. Relatively short time steps of $0.1 \mathrm{ps}$ were used in all simulations to ensure that the dynamical calculations avoid mathematically induced chaotic instabilities.

Off-axis electron holography provides the necessary high spatial resolution $(<10 \mathrm{~nm})$ to image the magnetic structure of vortices. ${ }^{13}$ Using a Philips CM300 field emission gun TEM, which accelerates the electrons with $300 \mathrm{kV}$, specimens were magnetized at an angle of $30^{\circ}$ with a magnetic field of about $2 \mathrm{~T}$ to create and pin the vortex domain wall close to the notch [Figs. 1(a) and 1(b)], as detailed in Ref. 16. A domain wall is situated adjacent to the constric-

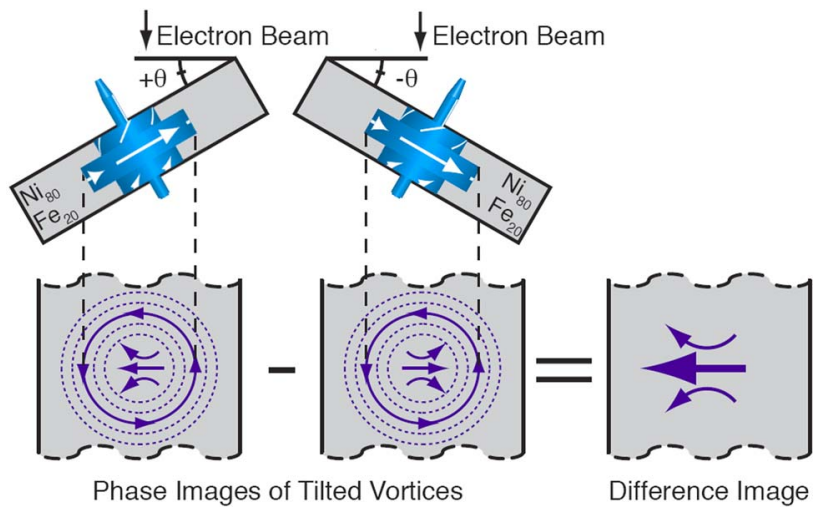

FIG. 2. (Color online) Schematic of the experimental procedure to extract the vortex core. The phase images show the projection of the magnetization within the sample at two equal but opposite tilts. During analysis these images are subtracted from each other (respectively divided), leaving only

trough distance of the phase images is a measure for the core width
the projection of the out-of-plane component.
Downloaded 25 Jun 2010 to 192.38.67.112. Redistribution subject to AlP license or copyright; see http://apl.aip.org/apl/copyrigh

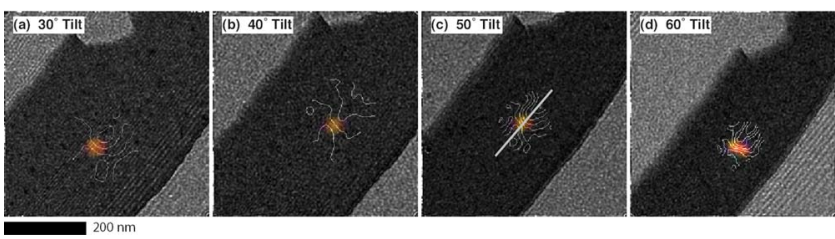

FIG. 3. (Color online) The equiphase contour lines of the out-of-plane component of a vortex in a notched permalloy nanowire are shown for different tilt angles of the sample [(a) $30^{\circ}$, (b) $40^{\circ}$, (c) $50^{\circ}$, and (d) $\left.60^{\circ}\right]$.

tion and the vortex spin structure with a vortex core in the center is visible [Figs. 1(b) and 1(c)]. In a standard electron holography image, only the in-plane magnetization is imaged. To observe the out-of-plane component of the vortex core, the sample is tilted around an axis from $-60^{\circ}$ to $-30^{\circ}$ and from $+30^{\circ}$ to $+60^{\circ}$ in $5^{\circ}$ steps [Fig. 1(c)-1(f)]. In principle, it would be enough to acquire two holograms at one positive and one negative tilt angle. However, the extra images are taken to ensure the signal is real and not a random artifact. At each angle, a specimen hologram and a reference hologram are acquired, while using a biprism voltage of $200 \mathrm{~V}$. The corresponding complex images of the negative and the positive tilting angles (e.g., $-60^{\circ}$ and $+60^{\circ}$ ) are subtracted, which allows the in-plane magnetic induction components to cancel out, leaving behind twice the value of the out-of-plane component (Fig. 2). During this procedure, the vortex cores in the two images must be aligned on top of each other with a high accuracy to obtain a measurable signal. If the images are misaligned, strong spurious signals are created.

After subtracting each pair of images, the same out-ofplane spin structure is visible in all of the images at the position of the vortex core (Fig. 3). The flux lines indicate the direction of the magnetization projection of the out-ofplane vortex core on the tilted plane of the image. Together with the known tilt direction, this yields the direction of the vortex core. The core position can also be determined from the in-plane induction map [Figs. 1(c)-1(f)]. The out-ofplane component is more readily observed at higher tilt angles because more of the core's stray field is projected into the plane of the image, as seen in Figs. 3(a)-3(d).

To determine the vortex core dimensions quantitatively, profiles along the line across the vortex core [see Fig. 3(c)] in the divided images (Fig. 3) are extracted. Those profiles are plotted in Fig. 4(a) representing the magnetic phase shift $\Phi_{\text {mag }}(x)=\int e / \hbar B\left(x^{\prime}\right) t \mathrm{dx}^{\prime}$, where $B$ is the magnetic induction,
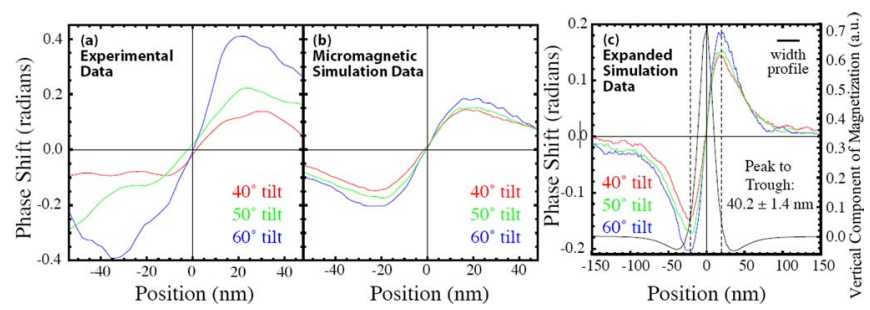

FIG. 4. (Color online) (a) Profiles across the vortex core of the divided phase images $\left[40^{\circ}\right.$ from Fig. 3(b), 50 ${ }^{\circ}$ from Fig. 3(c), $60^{\circ}$ from Fig. 3(d) $]$ are compared to (b) profiles extracted from phase images acquired from corresponding micromagnetic simulations at tilt angles $40^{\circ}, 50^{\circ}$, and $60^{\circ}$. (c) Expanded $( \pm 150 \mu \mathrm{m})$ simulated profiles. The out-of-plane component of magnetization is plotted for the vortex core indicating that the peak-totrough distance of the phase images is a measure for the core width 
$t$ the sample thickness, and $x$ a direction in the plane of the sample.

Micromagnetic simulations were carried out for comparison with the experimental observations. Integrated phase images were generated from simulations at tilts ranging from $30^{\circ}$ to $60^{\circ}$ and from $-30^{\circ}$ to $-60^{\circ}$ in $5^{\circ}$ steps. The mathematical difference between positive and negative tilt pairs (e.g., $+60^{\circ}$ and $-60^{\circ}$ tilts) was used to determine the morphology of the vortex core. Profiles across selected resulting phase difference images are shown in Fig. 4(b). Extended profiles are shown in Fig. 4(c).

The experimental profiles are similar to the simulated ones seen in Fig. 4. The most striking features of these profiles are the peaks and troughs. The amplitude of the profiles increases systematically with tilt angle. In contrast, the peakto-trough width which corresponds to the vortex width in the plane of the image, remains stable independent of the tilt angle. From all the traces an experimental value of $42 \pm 5 \mathrm{~nm}$ is found, which agrees well with the simulated values of $40.2 \pm 1.4 \mathrm{~nm}$.

In addition to the vortex width, also the experimental values of the phase shift for $40^{\circ}$ and $50^{\circ}$ tilt increasing from 0.1 to $0.2 \mathrm{rad}$ agree with the values from micromagnetic simulations. Quantitative difference between the simulation and the experiment for higher tilt angles are likely to be due to the imperfect tilt axis, misalignment, and artifacts during the image processing, resulting from a thicker effective substrate at a higher tilt angle.

A profile of the out-of-plane component of magnetization of the vortex core is shown together with the phase shifts [Fig. 4(c)]. The positions of the peaks and troughs of the phase difference profiles intersect the vertical component profile [vertical lines in Fig. 4(c)] at 10\% of the maximum value, indicating that the peak-to-trough width is a relatively accurate indicator of vortex core width. As expected, the center of the vortex shows the largest out-of-plane magnetization. Small negative components of magnetization are present on either side of the vortex, and are interpreted to be symmetric reactions to the demagnetizing field generated by the vortex core itself. ${ }^{2}$

Vortex core dimensions can also be extracted from measurements of the in-plane induction. We have taken profiles across the wire and the vortex core of the single images [see line in Fig. 1(e)]. Measurements on the profiles are carried out, as described in Ref. 13. The projected vortex core magnetization decreases like a cosine with increasing tilting angle and its width at $0^{\circ}$ tilt is extrapolated to be $54.8 \pm 11.7 \mathrm{~nm}$. The large errors obtained from measurements are due to noise around the vortex core which has no in-plane component. This shows that our direct measurements of the out-of-plane component are much more accurate and allow us to also determine the direction of the core.

In conclusion we have determined quantitatively the complete in-plane and out-of-plane spin structure of a vortex in a polycrystalline Permalloy nanostructure. The continuous change in magnetization across the center of a vortex is similar to that across a Bloch wall and, consequently, the width of these magnetic microstructures should also be of the same order of magnitude. The domain wall width has been defined in numerous ways in the literature. ${ }^{19}$ Domain wall width in thin films are calculated by Hubert and Schäfer ${ }^{19}$ using $W_{M}$ $=2 \sqrt{A / K}$ (proportional to exchange length) and it is this definition that we use here, where $A$ is the exchange stiffness constant $(\mu \mathrm{erg} / \mathrm{cm})$ and $K$ is an anisotropy coefficient term, which in the case of Permalloy is the anisotropy coefficient of stray field energy density, $K_{d}=M_{s}^{2} / 2 \mu_{0}$. $M_{s}$ is the saturation magnetization $\left(\mathrm{emu} \mathrm{cm}^{-3}\right.$ ) and $\mu_{0}$ is the permeability of free space, which in cgs units has a value of unity. The width of the vortex core observed in the holograms and the micromagnetic simulations is very similar to the expected width of a Bloch wall in Permalloy thin film, which varies from 36 to $50 \mathrm{~nm}$ depending on different values of the exchange constant $A$, which is not precisely known. ${ }^{20-22}$

The authors acknowledge support by the Deutsche Forschungsgemeinschaft (SFB 513), Landesstiftung Baden Württemberg, and the EPSRC (UK). One of the authors (F.J.) acknowledges support by the "Studienstiftung des Deutschen Volkes," D.B. acknowledges support by the European Union (Interreg III A program), R.E.D.B. acknowledges support by the Royal Society, and J.M.F. thanks NERC (UK) for financial support.

${ }^{1}$ S. S. P. Parkin/IBM U.S. Patent No. 10/984,055 (9 November 2005).

${ }^{2}$ A. Wachowiak, J. Wiebe, M. Bode, O. Pietzsch, M. Morgenstern, and R. Wiesendanger, Science 298, 577 (2002).

${ }^{3}$ T. Shinjo, T. Okuno, R. Hassdorf, K. Shigeto, and T. Ono, Science 289, 930 (2000).

${ }^{4}$ A. Yamasaki, W. Wulfhekel, R. Hertel, S. Suga, and J. Kirschner, Phys. Rev. Lett. 91, 127201 (2003).

${ }^{5}$ H. Kohno, G. Tatara, J. Shibata, and Y. Suzuki, J. Magn. Magn. Mater. 310, 2020 (2007).

${ }^{6}$ B. Van Waeyenberge, A. Puzic, H. Stoll, K. W. Chou, T. Tyliszczak, R. Hertel, M. Fähnle, H. Brückl, K. Rott, G. Reiss, I. Neudecker, D. Weiss, C. H. Back, and G. Schütz, Nature (London) 444, 05240 (2006).

${ }^{7}$ A. Thiaville, Y. Nakatani, J. Miltat, and Y. Suzuki, Europhys. Lett. 69, 990 (2005).

${ }^{8}$ A. Yamaguchi, T. Ono, S. Nasu, K. Miyake, K. Mibu, and T. Shinjo, Phys. Rev. Lett. 92, 077205 (2004).

${ }^{9}$ M. Kläui, C. A. F. Vaz, J. A. C. Bland, W. Wernsdorfer, G. Faini, E. Cambril, L. J. Heyderman, F. Nolting, and U. Rüdiger, Phys. Rev. Lett. 94, 106601 (2005).

${ }^{10}$ F. Junginger, M. Kläui, D. Backes, U. Rüdiger, T. Kasama, R. E. DuninBorkowski, L. J. Heyderman, C. A. F. Vaz, and J. A. C. Bland, Appl. Phys. Lett. 90, 132506 (2007).

${ }^{11}$ M. Kläui, P.-O. Jubert, R. Allenspach, A. Bischof, J. A. C. Bland, G. Faini, U. Rüdiger, C. A. F. Vaz, L. Vila, and C. Vouille, Phys. Rev. Lett. 95, 026601 (2005).

${ }^{12}$ M. Laufenberg, D. Backes, W. Bührer, D. Bedau, M. Kläui, U. Rüdiger, C. A. F. Vaz, J. A. C. Bland, L. J. Heyderman, F. Nolting, S. Cherifi, A. Locatelli, R. Belkhou, and S. Heun, Appl. Phys. Lett. 88, 052507 (2006).

${ }^{13}$ R. E. Dunin-Borkowski, M. R. McCartney, and D. J. Smith, Encyclopaedia of Nanoscience and Nanotechnology, edited by H. S. Nalwa (American Scientific, Stevenson Ranch, CA, 2004), Vol. 3, pp. 91-100.

${ }^{14}$ G. Lai, T. Hirayama, A. Fukuhara, K. Ishizuka, T. Tanji, and A. Tonomura, J. Appl. Phys. 75, 4593 (1994).

${ }^{15}$ D. Backes, L. J. Heyderman, C. David, R. Schäublin, M. Kläui, H. Ehrke, U. Rüdiger, C. A. F. Vaz, J. A. C. Bland, T. Kasama, and R. E. DuninBorkowski, Microelectron. Eng. 83, 1726 (2006).

${ }^{16}$ M. Kläui, H. Ehrke, U. Rüdiger, T. Kasama, R. E. Dunin-Borkowski, D. Backes, L. J. Heyderman, C. A. F. Vaz, J. A. C. Bland, G. Faini, E. Cambril, and W. Wernsdorfer, Appl. Phys. Lett. 87, 102509 (2005).

${ }^{17}$ M. R. Scheinfein, v2.50 ed., 2006 (http://llgmicro.home.mindspring.com).

${ }^{18}$ P. Barpanda, T. Kasama, R. E. Dunin-Borkowski, M. R. Scheinfein, and A. S. Arrott, J. Appl. Phys. 99, 08 G103 (2006).

${ }^{19}$ A. Hubert and R. Schäfer, Magnetic Domains: The Analysis of Magnetic Microstructures, 1st ed. (Springer, Heidelberg, 1998), p. 219.

${ }^{20}$ F. B. Humphrey and M. Redjdal, J. Magn. Magn. Mater. 133, 11 (1994).

${ }^{21}$ G. M. Sandler, H. N. Bertram, T. J. Silva, and T. M. Crawford, J. Appl. Phys. 85, 5080 (1999).

${ }^{22}$ N. Kikuchi, S. Okamoto, O. Kitakami, Y. Shimada, S. G. Kim, Y. Otani, and K. Fukamichi, IEEE Trans. Magn. 37, 2082 (2001). 